\title{
Functional vascular imaging by Photoacoustic Microscopy (PAM) and its biomedical application
}

Renzhe Bi, Ghayathri Balasundaram, Dinish U.S., Seungwan Jeon, Toru Imai, et al.

Renzhe Bi, Ghayathri Balasundaram, Dinish U.S., Seungwan Jeon, Toru Imai, Yang Pu, Lai Guan Ng, Chulhong Kim, Lihong Wang, Malini Olivo, "Functional vascular imaging by Photoacoustic Microscopy (PAM) and its biomedical application," Proc. SPIE 10873, Optical Biopsy XVII: Toward RealTime Spectroscopic Imaging and Diagnosis, 108730B (4 March 2019); doi: $10.1117 / 12.2507355$

SPIE. Event: SPIE BiOS, 2019, San Francisco, California, United States 


\title{
Functional vascular imaging by Photoacoustic Microscopy (PAM) and its biomedical application
}

\author{
Renzhe Bi ${ }^{\mathrm{a}}$, Ghayathri Balasundaram ${ }^{\mathrm{a}}$, U. S Dinish ${ }^{\mathrm{a}}$, Seungwan Jeon ${ }^{\mathrm{b}}$, Toru Imai ${ }^{\mathrm{c}}$, Yang Pu ${ }^{\mathrm{d}}$, Lai \\ Guan $\mathrm{Ng}^{\mathrm{e}}$, Chulhong Kim ${ }^{\mathrm{b}}$, Lihong Wang ${ }^{\mathrm{c}}$, Malini Olivo ${ }^{\mathrm{a}}$ \\ ${ }^{\mathrm{a}}$ Singapore Bioimaging Consortium, 11 Biopolis Way, Singapore 138667 \\ ${ }^{b}$ Department of Creative IT Engineering, Pohang University of Science and Technology, 77 \\ Cheongam-ro, Nam-gu, Pohang, Gyeongbuk, Republic of Korea 37673 \\ ${ }^{c}$ Caltech Optical Imaging Laboratory, Andrew and Peggy Cherng Department of Medical \\ Engineering and Department of Electrical Engineering, California Institute of Technology, 1200 \\ East California Boulevard, Pasadena, CA 91125, USA \\ ${ }^{\mathrm{d}}$ MicroPhotoAcoustics Inc., 105 Comac Street, Ronkonkoma, NY USA 11779 \\ ${ }^{\mathrm{e}}$ Singapore Immunology Network (SIgN), Agency for Science Technology and Research \\ (A*STAR), Singapore
}

\begin{abstract}
The hemodynamics and oxygen saturation status of vascular are very important biomarkers for disease, such as brain glioma tumor and ischemia-reperfusion ulcer. Therefore, a high spatial resolution imaging tool for vascular imaging is demanded. Conventional optical imaging modalities, including confocal microscopy and two-photon microscopy, require external contrast agent to image blood vessels and are not sensitive to oxygen saturation. The development of photoacoustic microscopy provides a contrast-free, high-spatial resolution and functional vascular imaging tool. It's gaining more and more popularity in biomedical research. In this paper, we introduce a dual-wavelength opticalresolution photoacoustic microscopy (OR-PAM) system for functional imaging of vasculature. This system has demonstrated its application in brain glioma tumor imaging, as well as skin ischemia-reperfusion imaging.
\end{abstract}

Keywords: Photoacoustic microscopy, functional imaging, biomedical imaging, vascular imaging

\section{INTRODUCTION}

The hemodynamics and oxygen saturation status of vascular are very important biomarkers for disease [1-5], such as brain glioma tumor [6] and ischemia-reperfusion ulcer [7]. Therefore, a high spatial resolution imaging tool for vascular imaging is demanded. Conventional optical imaging modalities, including confocal microscopy and two-photon microscopy, require external contrast agent injection to image blood vessels [7-10]. Besides, they can only image the vascular outline but not the oxygen saturation inside the vessels.

Photoacoustic imaging (PAI) takes advantage of optical illumination and ultrasound detection, which enables contrastfree vascular imaging in deeper tissue [11-14]. By using optical illumination and taking advantage of optical absorption contrast, PAI is able to distinguish the main endogenous chromophores in biological tissue, including melanin, oxyhemoglobin, deoxy-hemoglobin, water and lipid by using multiple lasers at different wavelengths [15]. Meanwhile, various exogenous contrast agents have been developed to assist PAI to achieve more specific measurement [16,17]. Since biological tissue is highly scattering medium [18-21], optical signal easily gets scattered and thus attenuated $[22,23]$. The imaging penetration has been always one of the major challenges of pure-optical imaging methods. PAI employs ultrasound detection instead of optical detection. The scattering coefficient of ultrasound signal in biological tissue is $2-3$ orders smaller than that of the optical signal $[24,25]$. Consequently, the imaging depth of PAI is much

* Malini_olivo@sbic.a-star.edu.sg Tel: 65-64788745 Fax:65-64789957 
higher than conventional optical imaging methods.

Because of the advantages of contrast-free measurement and deeper penetration, PAI is widely utilized in biomedical applications which involve imaging and monitoring of the vasculature $[6,26,27]$. In this paper, we introduce a dualwavelength water-immersible-MEMS based optical-resolution photoacoustic microscopy (OR-PAM) system for high spatial resolution functional imaging of blood vessels. The mechanical scanning OR-PAM system has been used for brain glioma tumor imaging and the treatment evaluation of combretastatin A4 phosphate (CA4P), an FDA approved anticancer drug [6]. Thanks to the high spatial resolution, OR-PAM could map the treatment response of every individual blood vessel. The study of glioma tumor monitoring has been reported in detail in other papers.

The upgraded MEMS-OR-PAM system has a high A-line scanning speed (>10 kHz), which enables continuous imaging of some gradual-changing physiological phenomenon. Two individual nano-second pulsed laser $(532 \mathrm{~nm}$ and $559 \mathrm{~nm})$ are combined into a single beam. Fast wavelength switching can be achieved by using two lasers. Therefore, for each Aline scanning, the signal excited by both lasers are acquired. By using this method, the total imaging time of dual wavelengths is the same as single wavelength imaging. By applying a spectrum unmixing algorithm, the oxygen saturation image can be generated from the raw images of the two wavelengths.

The fast functional-imaging enables continuously monitoring of the vascular hemodynamic change. Vascular hemodynamic monitoring is critical for understanding ischemia-reperfusion (IR), which is the main cause of bedsore. OR-PAM provides a non-invasive and high spatial resolution method to monitor the vascular during the reperfusion period. For the first time, the revascularization process can be imaged without any contrast agent injection. Moreover, the oxygen saturation of the veins can be used as a biomarker to understand the cause of the ulcer formation. Begin the Introduction two lines below the Keywords. The manuscript should not have headers, footers, or page numbers. It should be in a one-column format. References are often noted in the text ${ }^{1}$ and cited at the end of the paper.

\section{IMAGING SYSTEM}

One of the most important components of a conventional OR-PAM system is the photoacoustic (PA) sensor. A PA sensor has an optical-acoustic combiner to deliver the excitation light source and collect the stimulated ultrasound signal, as shown in Fig 1 (a). A two-dimensional mechanical motor system drives the PA sensor to scan the sample in x-y plane, as shown in Fig 1 (b).
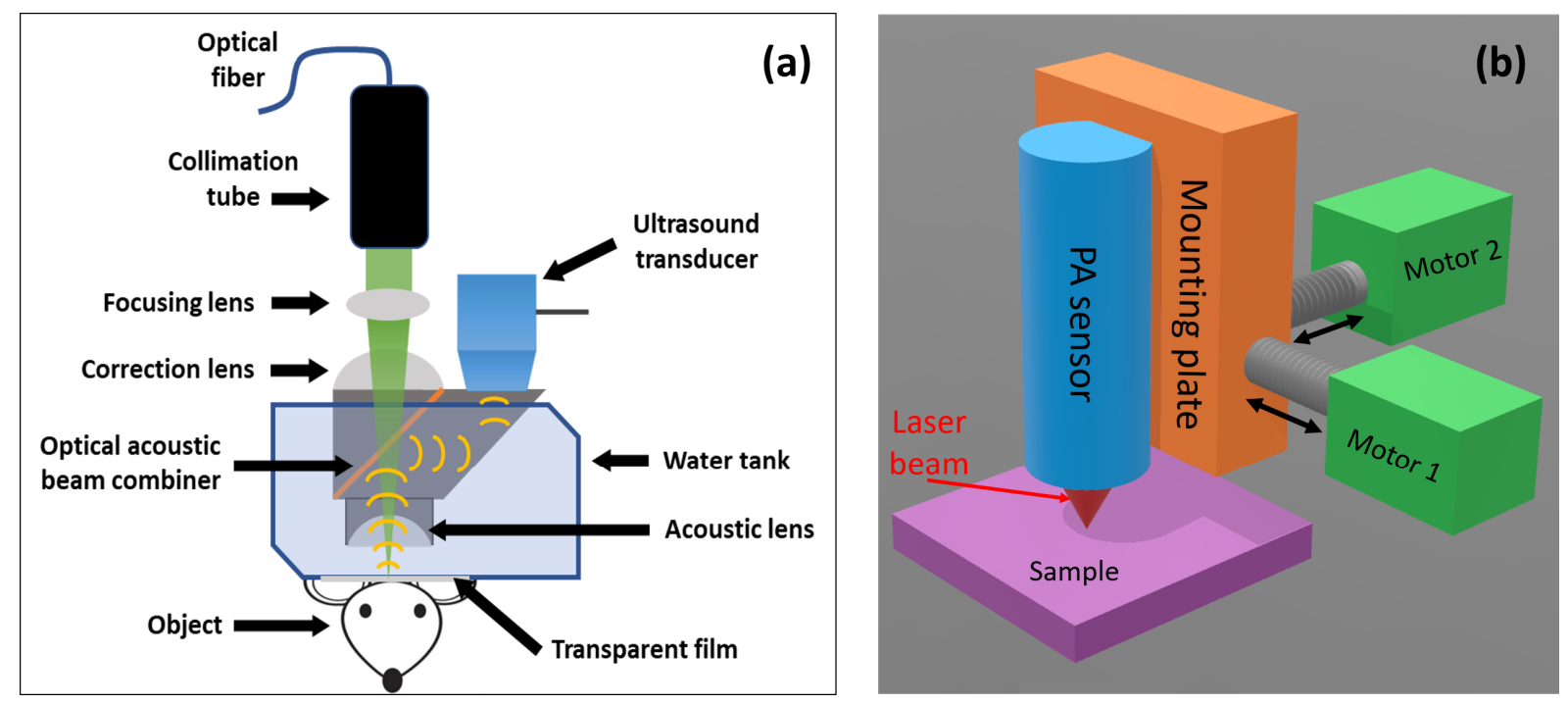

Figure 1. (a) Schematic of a typical PA sensor in OR-PAM system. An optical-acoustic beam combiner aligns the focused optical beam and the focused ultrasound detection beam confocally. The confocal configuration guarantees the best signal-to-noise ratio. (b) Schematic of the two-dimensional mechanical motor system for scanning. The PA sensor is mounted on the motor system and driven across the entire $x-y$ plane. 
The lateral resolution of an OR-PAM image is determined by the size of optical focus. By focusing the optical beam, very high spatial resolution can be achieved. Typical OR-PAM resolution varies from $0.3 \mu \mathrm{m}$ to $10 \mu \mathrm{m}$, depending on the excitation wavelength and the numerical aperture (NA) of the optical focusing lens. Here we present a resolution characterization result of a mechanical scanning OR-PAM system in Fig 2. The resolution target follows the 1951 USAF standard. In group 6, the width of the lines of element 4 is $5.52 \mu \mathrm{m}$. These lines can be distinguished as shown in the plot of Fig 2. Since the size of capillaries is around $10 \mu \mathrm{m}$, this resolution is sufficient for any vascular imaging requirement.
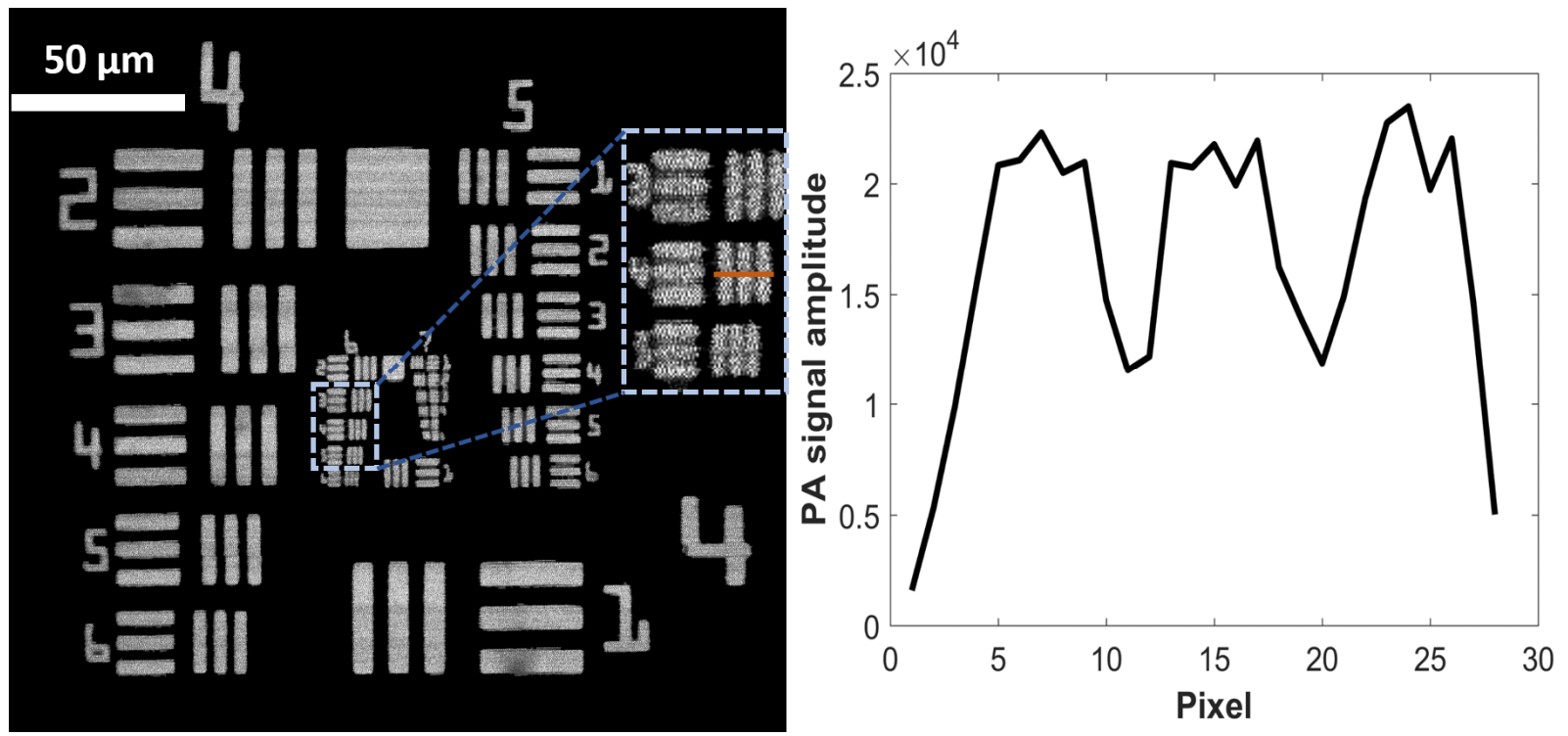

Figure 2. Resolution characterization of a mechanical scanning OR-PAM system using a 1951 USAF resolution target. In group 6 , the line width of element 4 is $5.52 \mu \mathrm{m}$.

Although conventional mechanical scanning OR-PAM system provides high spatial resolution, contrast-free vascular imaging and functional imaging capability, the imaging speed is a big limitation for real biomedical application. Typical acquisition time for one image is from 10 min to a couple of hours, depending on the scanning size. The main challenge is the scanning speed of the mechanical motor, which is normally limited within $20 \mathrm{~mm} / \mathrm{s}$.

To improve the imaging speed of OR-PAM system, there are several different methods being explored, including voicecoil scanning stage, hexagon scanner and microelectromechanical system (MEMS). This paper introduces a fast scanning dual-wavelength OR-PAM system based on a water-immersible MEMS scanner and its application in continuous monitoring of the skin ischemia reperfusion process.

The schematic of the dual-wavelength MEMS-OR-PAM system is demonstrated in Fig 3. The two lasers (532 nm and $559 \mathrm{~nm}$ ) are combined into one single beam. Then the beam is collimated and received by a fiber collimator. An iris with tuneable aperture is used to control the light intensity. The fiber then delivers the laser into the PA sensor. Along the optical path in the PA sensor, a 95 (transmission):5 (reflection) beam splitter is used to spare a small portion of the light into a photodiode. During imaging, the photodiode records every laser pulse intensity for normalization purpose. A water-immersible MEMS scanner reflects the optical beam onto the sample and acoustic signal into the ultrasound transducer. The MEMS scanner works in single dimension. A mechanical motor works at the diagonal direction of the MEMS scanner. The combination between MEMS and mechanical motor guarantees fast imaging speed and imaging field of view. A PC with LabVIEW controls the lasers, MEMS, mechanical motor and the acquisition of ultrasound transducer. 


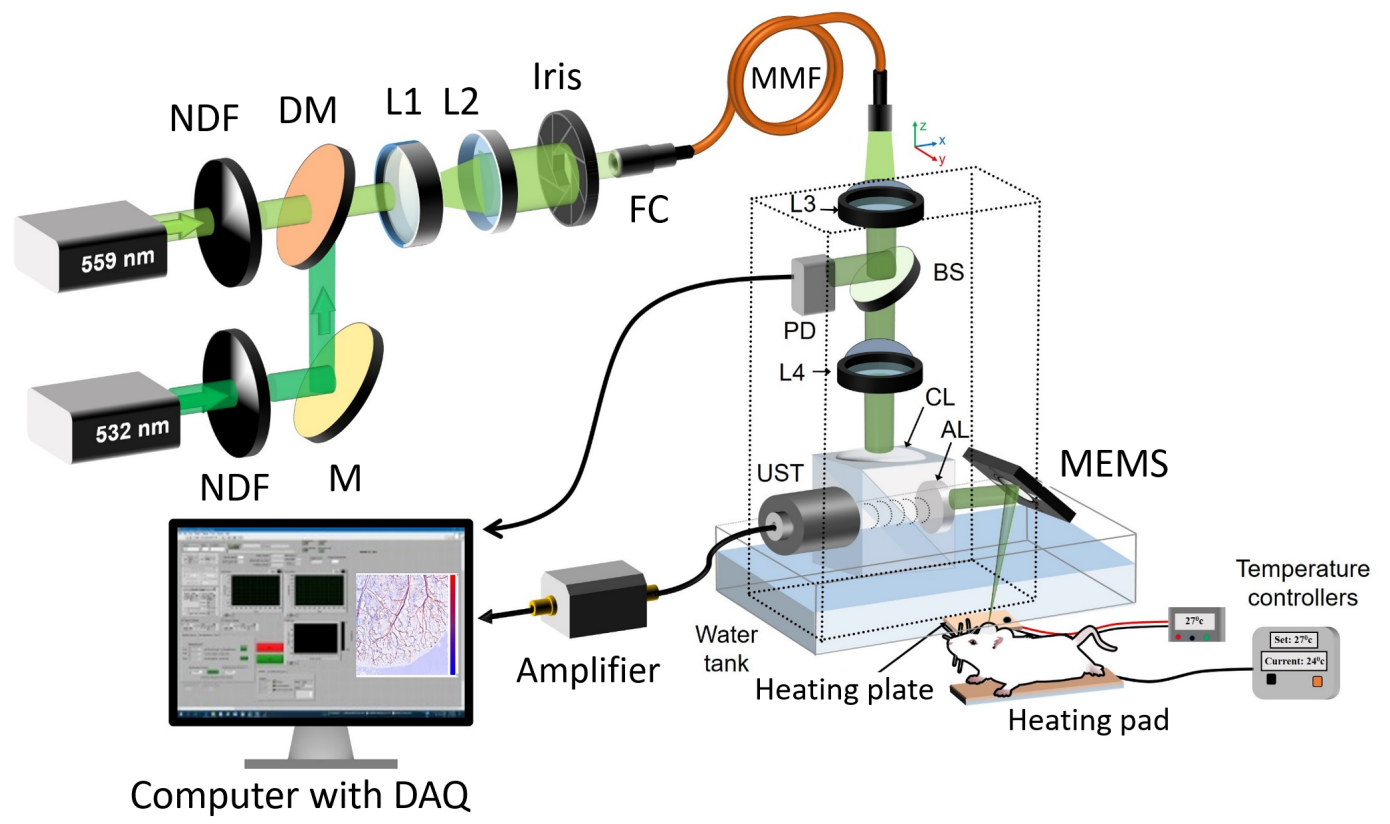

Figure 3. Schematic of dual-wavelength MEMS-OR-PAM imaging system. NDF: neutral density filter, DM: dichroic mirror, L1, L2, L3, L4: lens, FC: fiber collimator, PD: photodiode, BS: beam splitter, UST: ultrasound transducer, AL: acoustic lens.

\section{RESULT}

The ischemia was induced to the mouse ear skin by clamping two magnets for 1.5 hours. Before the release of the magnets, the mice were anaesthetized by ketamine-xylazine $\left(15 \mathrm{mg} \mathrm{mL}^{-1}\right.$ ketamine mixed with $1 \mathrm{mg} \mathrm{mL}^{-1}$ xylazine; $8 \mu \mathrm{L}$ $\mathrm{g}^{-1}$ bodyweight) and placed on the imaging stage of the MEMS-OR-PAM system. Immediately after the release of the magnets, the water tank was installed on top of ear skin. Then the bottom part of the PA sensor was immersed in the water tank.

Dual-wavelength MEMS-OR-PAM system was used to image the ischemic ear skin continuously with a time interval of $4 \mathrm{~min}$. The entire imaging period lasted for about 2 hours, within which the vascular network re-vascularized from the ischemic status. The vascular images at several time points along the reperfusion process are shown in Fig 4.

Comparing with the unaffected tissue on the left side, the ischemic tissue had very little blood signal and broken blood vessels in the beginning of reperfusion (Fig $4, \mathrm{t}=0$ ). The big vessels in the ischemic tissue gradually recover, especially the arteries. The capillaries followed the big vessels and got reperfused. At the end of the reperfusion (Fig 4, $\mathrm{t}=128 \mathrm{~min}$ ), the vascular network fully connected with significant amount of blood signals.

The entire reperfusion could be captured continuously by the dual-wavelength MEMS-OR-PAM system at a time interval of 4 min. The detailed changes of the vascular network will provide significant help to biological studies. 


\section{Reperfusion}

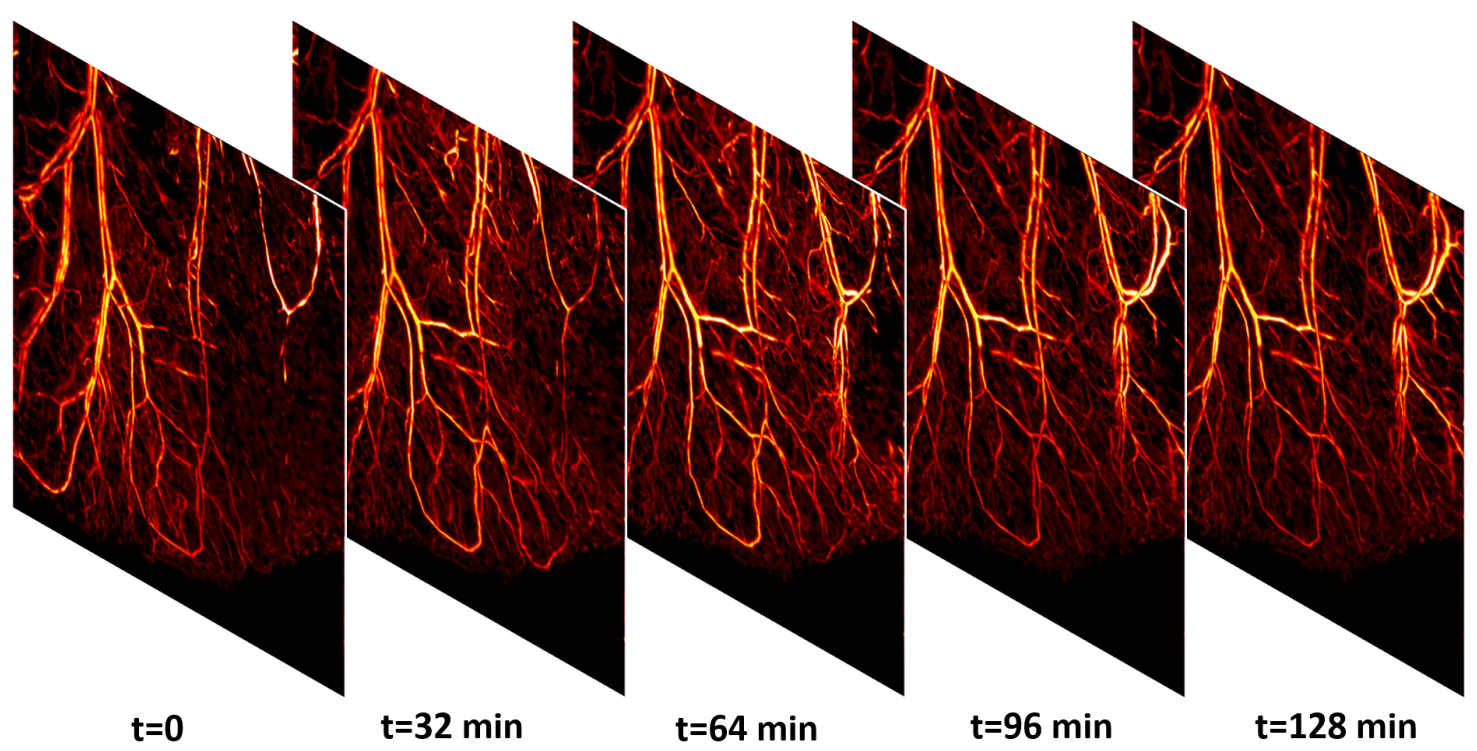

Figure 4. Vascular images of the re-perfusing ear skin at different time points with a time interval of $32 \mathrm{~min}$. At $\mathrm{t}=0$, the ischemic region has only very little blood signal and the blood vessels are broken. The big vessels start to join together and followed by the capillaries. At the end of reperfusion, all the blood vessels are back to normal status.

\section{CONCLUSION AND DISCUSSION}

Our MEMS-OR-PAM system provides fast dual-wavelength imaging capability, which enables continuous monitoring of gradual-changing biological phenomenon, such as ischemia-reperfusion. PAM has been gaining popularity in biological applications, such as tumor monitoring and vascular diseases. We envision that it will play an important role in biomedical environment in the future.

\section{REFERENCES}

[1] H. R. Weiss, J. Grayson, X. Liu, S. Barsoum, H. Shah, and O. Z. Chi, "Cerebral Ischemia and Reperfusion Increases the Heterogeneity of Local Oxygen Supply / Consumption Balance," Stroke 44, 2553-2558 (2013).

[2] F. Liao, S. Burns, and Y.-K. Jan, "Skin blood flow dynamics and its role in pressure ulcers," J. Tissue Viability 22, 25-36 (2013).

[3] J. Dong, H. J. Toh, P. S. P. Thong, C. S. Tee, R. Bi, K.-C. Soo, and K. Lee, "Hemodynamic monitoring of Chlorin e6-mediated photodynamic therapy using diffuse optical measurements," J. Photochem. Photobiol. B Biol. 140, 163-172 (2014).

[4] Sordillo, L. A., Pu, Y., Pratavieira, S., Budansky, Y. and Alfano, R. R., "Deep optical imaging of tissue using the second and third near-infrared spectral windows," J. Biomed. Opt. 19, 056004 (2014)

[5] Pu, Y., Wang, W. B., Xu, M., Eastham, J., Tang, G. C. and Alfano, R. R., "Characterization and threedimensional localization of cancerous prostate tissue using backscattering scanning polarization imaging and independent component analysis," J. Biomed. Opt. 17(8), 081419 (2012).

[6] Bi, R., Balasundaram, G., Jeon, S., Tay, H. C., Pu, Y., Li, X., Moothanchery, M., Kim, C., and Olivo, M, "Front Cover: Photoacoustic microscopy for evaluating combretastatin A4 phosphate induced vascular disruption in orthotopic glioma (J. Biophotonics 10/2018)," J. Biophotonics 11, (2018). 
[7] C. C. Goh, M. Evrard, S. Z. Chong, Y. Tan, L. D. L. Tan, K. W. W. Teng, W. Weninger, D. L. Becker, H. L. Tey, E. W. Newell, B. Liu, and L. G. Ng, "The impact of ischemia-reperfusion injuries on skin resident murine dendritic cells," Eur. J. Immunol. 1-6 (2018).

[8] C. C. Goh, J. L. Li, S. Devi, N. Bakocevic, P. See, A. Larbi, W. Weninger, F. Ginhoux, V. Angeli, and L. G. Ng, "Real-Time Imaging of Dendritic Cell Responses to Sterile Tissue Injury," J. Invest. Dermatol. 135, 1181-1184 (2015).

[9] Pu, Y., Tang, R., Xue, J., Wang, W. B., Xu, B., and Achilefu, S., "Synthesis of dye conjugates to visualize the cancer cells using fluorescence microscopy," Appl. Opt. 53(11), 2345 - 2351 (2014).

[10] Pu, Y., Shi, L., Pratavieira, S. and R. Alfano, R. R., "Two-photon excitation microscopy using the second singlet state of fluorescent agents within the 'tissue optical window'," J. Appl. Phys. 114(15), 153102 (2013).

[11]Bi, R., Ma, Q., Mo, H., Olivo, M., and Pu, Y., "Chapter 8 - Optical resolution photoacoustic microscope of brain imaging in small animal model using solid state nanosecond laser," in R. R. Alfano (Eds.) [Neurophotonics and biomedical spectroscopy], Elsevier Ltd., 159-187 (2018).

[12] Moothanchery, M., Bi, R., Kim, J. Y., Jeon, S., Kim, C., Olivo, M., "Optical resolution photoacoustic microscopy based on multimode fibers," Biomed Opt. Express. 9(3), 1190-1197 (2018).

[13] L. Lin, P. Hu, J. Shi, C. M. Appleton, K. Maslov, L. Li, R. Zhang, and L. V Wang, "Single-breath-hold photoacoustic computed tomography of the breast," Nat. Commun. 9, 2352 (2018).

[14] S. Hu, K. Maslov, and L. V. Wang, "Second-generation optical-resolution photoacoustic microscopy with improved sensitivity and speed," Opt. Lett. 36, 1134 (2011).

[15] V. Ntziachristos and D. Razansky, "Molecular imaging by means of multispectral optoacoustic tomography (MSOT)," Chem. Rev. 110, 2783-2794 (2010).

[16]D. Su, C. L. Teoh, S.-J. Park, J.-J. Kim, A. Samanta, R. Bi, U. S. Dinish, M. Olivo, M. Piantino, F. Louis, M. Matsusaki, S. S. Kim, M. A. Bae, and Y.-T. Chang, "Seeing Elastin: A Near-Infrared Zwitterionic Fluorescent Probe for In Vivo Elastin Imaging," Chem 4, 1128-1138 (2018).

[17] Pu, Y., Wang, W.B., Das, B. B., Achilefu, S. and Alfano, R. R., "Time-resolved fluorescence polarization dynamics and optical imaging of Cytate: a prostate cancer receptor-targeted contrast agent," Appl. Opt. 47, 2281-2289 (2008).

[18]Bi, B., Dong, J., and Lee, K., "Deep tissue flowmetry based on diffuse speckle contrast analysis," Opt. Lett. 38, 1401-1403 (2013).

[19] Bi, R., Dong, J., Poh, C. L., and Lee, K., "Optical methods for blood perfusion measurement - theoretical comparison among four different modalities," J. Opt. Soc. Am. A 32, 860-866 (2015).

[20] Bi, R., Dong, J., and Lee, K., "Multi-channel deep tissue flowmetry based on temporal diffuse speckle contrast analysis," Opt. Express. 21, 22854-22861 (2013).

[21] J. Dong, R. Bi, J. H. Ho, P. S. P. Thong, K.-C. Soo, and K. Lee, "Diffuse correlation spectroscopy with a fast Fourier transform-based software autocorrelator," J. Biomed. Opt. 17, 97001-97004 (2012).

[22] Pu, Y., Wang, W. B., AL-Rubaiee, M., Gayen, S. K., and Xu, M., "Determination of optical coefficients and fractal dimensional parameters of cancerous and normal prostate tissues," Appl. Spectrosc. 66(7), 828834 (2012).

[23] Pu, Y., Wang, W. B., Tang, G. C., Zeng, F., Achilefu, S., Vitenson, J. H., Sawczuk, I., Peters, S., Lombardo, J. M. and Alfano, R. R., "Spectral polarization imaging of human prostate cancer tissue using near-infrared receptor-targeted contrast agent," Technol. Cancer Res. Treat. (TCRT) 4, 429-436 (2005).

[24] J. Kim, S. Park, Y. Jung, S. Chang, J. Park, Y. Zhang, J. F. Lovell, and C. Kim, "Programmable Real-time Clinical Photoacoustic and Ultrasound Imaging System," Sci. Rep. 6, 35137 (2016).

[25] L. V. Wang, "Multiscale photoacoustic microscopy and computed tomography," Nat. Photonics 3, 503-509 (2009).

[26] J. Kim, Y. H. Kim, B. Park, H. M. Seo, C. H. Bang, G. S. Park, Y. M. Park, J. W. Rhie, J. H. Lee, and C. Kim, "Multispectral ex vivo photoacoustic imaging of cutaneous melanoma for better selection of the excision margin," Br. J. Dermatol. 0-3 (2018).

[27] A. B. E. Attia, S. Y. Chuah, D. Razansky, C. J. H. Ho, P. Malempati, U. S. Dinish, R. Bi, C. Y. Fu, S. J. Ford, J. S. S. Lee, M. W. P. Tan, M. Olivo, and S. T. G. Thng, "Noninvasive real-time characterization of non-melanoma skin cancers with handheld optoacoustic probes," Photoacoustics 7, 20-26 (2017). 